\title{
NONCOMMUTATIVE DEFORMATION AND A TOPOLOGICAL NATURE OF KOITER SINGULARITY
}

\author{
TRINH VAN KHOA \\ Hanoi Architectural U̦iviversity
}

\begin{abstract}
In this paper we constructed the model of noncommutative plastic deformation and give the proof of Koiter hypothesis. We showed, that the occurrence of Koiter singularity has topological reasons and the number of Koiter singularities - is the topological Pontriagin number.
\end{abstract}

\section{INTRODUCTION}

The mechanism for planes of atoms shear in a crystal relative to one another is used when describing the process of plastic deformation in a simple case. The elastic limit is defined as $\tau_{c r}=\frac{G a}{2 \pi d}$, where $a$ is the interatomic distance, $d$ is the distance between the glide planes, and therefore the value of $\tau_{c r}$ must be about an order of magnitude less than that of the shear modulus $G$. The experimental data [15] have shown, however, that for tin $G=1.9 \times 10^{11}$ dyne $/ \mathrm{cm}^{2}$, while $\tau \cong 13 \times 10^{6}$ dyne $/ \mathrm{cm}^{2}$, for silver the corresponding values are $2.8 \times 10^{11}$ and $6 \times 10^{6}$, for aluminium, $2.5 \times 10^{11}$ and $4 \times 10^{11} \mathrm{dyne} / \mathrm{cm}^{2}$. It should be noted that a more exact account of the arrangement of planes of atoms in shear yields an estimate $\tau_{c r} \sim G / 30$, and this exceeds the experimentally found value by several orders of magnitude. The reason for such a phenomenon is the coherence of rearrangements of the crystal structure. Therefore to describe plastic deformation, it is worthwhile to use the phenomenological approach accepted in continuum mechanics.

From the point of view of phenomenology, till now there are many models of plastic deformation. Almost in all monographs, for example [1-3, 13], the following models are considered: sliding, regular, current, singular, analytical and deformation plasticity. The determinant ratio in deformation is a major problem, which was submitted for discussion. The most simple kind was presented in the form of Hencky-Nadai:

$$
\begin{gathered}
\sigma_{i j}=2 G_{s}(T) e_{i j} \quad \text { at } \quad T=\bar{T}, \\
d \sigma_{i j}=2 G d e_{i j} \quad \text { at } \quad T<\bar{T},
\end{gathered}
$$

where $d \sigma_{i j}, e_{i j}$-deviator of a stress and deformation, $G_{s}$ - current shear modulus, $G$ - the elastic shear modulus, $T$ - intensity tangent stress, $\bar{T}$ - the maximal value $T$ for a history of loading pre-eminent some value $T_{0}$. In singularity model it looks like

$$
\mathrm{de}_{i j}=\sum_{n=1}^{N} d f_{n} \frac{\partial f_{n}}{\partial \sigma_{i j}},
$$

where $f_{n}$ - the current limiting surface. On the appropriate surface $f_{n}$ performed by loading, then $d f_{n} \neq 0$ i.e.

$$
\left(\frac{\partial f_{n}}{\partial \sigma_{i j}}\right) d \sigma_{i j} \geq 0 .
$$


In Ilyushin model it has the kind

$$
\sigma_{i j}=\sum_{n} A_{n} \frac{d^{n} e_{i j}}{d e^{n}} ; e=\int \sqrt{d e_{\alpha} d e_{\alpha}}
$$

However, if we use the concept of deformation potential as in [1-3,10-11], by definition of function $H$, we shall receive following determining ratio

$$
\sigma_{i j}=\frac{\partial H}{\partial e_{i j}} .
$$

In our model [6], we consider process of plastic deformation as process of phase transition, potential of deformation (or the free energy) plays a role of Hamilton function or an action of system. So, if we admit Hamilton function as $H=\alpha e^{2}+\beta e^{4}+\ldots$, where $\alpha, \beta$ - factors of elasticity, $e$ - deformation tensor, that as a first approximation we shall receive the formulas (1.1) (1.2) (1.3) (if $H$ is expressed through the metrics of spaces). Roughly speaking, the form (1.4) is general determining ratio for process of plastic deformation.

The present tense one develops a new idea to formulate the plastic theory. In the papers $[5,6]$ we developed a model of the crack in the vicinity of coherence (phase transition). More generally, we can consider this vicinity as a thermodynamical system far from equilibrium. Then we come to the problem of spontaneous breaking of structure. It is clear, we want to consider the plastic structure. Here the problem of valuation of the probability for spontaneous breaking of structure is arrived. It was solved with the aid of identification of deformation trajectory with a knot. So inner geometry of deformation and invariant of knot is interconnected. Under such approach we can construct a model of plastic deformation with more deep structure of geometry, in which topological characteristic plays a decision role [7]. In paper [18] Ch. Tsakmakis gives description of plastic anisotropy effects at large deformation, in which restrictions imposed by the second law and the postulate of Ilyushin. However, within the framework of our noncommutative model of plastic deformation when using strain-potential formalism, this postulate would become a theorem which could be proved [7].

All of model of plastic deformation bases on the hypothesis of the existence of limit surface of deformation. However, any model of plastic deformation with smooth limit surface of deformation has one specific feature - it is weak apprehensibility toward the break of deformation trajectory. To correct this lack W.T. Koiter submitted for consideration a hypothesis of existence of $n$ singularities on the limit surface of deformation [14]. Could this Koiter's hypothesis be correct or not? If it is correct, then what is the nature of Koiter's singularity? We try to solve these problems consistently. For this purpose we should construct a more general model of deformation. It means that, we should construct the model of plastic deformation based on the noncommutative nature of deformation space and the fluctuation characteristic of deformation field in process of fracture.

\section{MODEL OF PLASTIC DEFORMATION}

As it is known, all experimental data have shown, that in a plastic status the stress and deformation is strongly fluctuative. The pressure - deformation diagram $\sigma=f(e)$ is similar to the pressure - volume diagram $P=f(V)$ for liquid [8]. So, it is possible to 
consider a process of deformation as a process of phase transition. More general, it is a process of formation of structure [7]. The density of distribution of transition probability to plasticity is a solution of the Fokker-Planck equation. However, if using potential character of process of deformation it is possible instead of the Fokker-Planck equation we apply Schrodinger equation. In this approach the deformation tensor is chosen in the role of order parameter. In the work [1] A. A. Ilyusin has presented the concept of deformation trajectory in space $E_{5}$. As a matter of fact, the space $E_{5}$ is a fiber in the deformation bundle [7]. Further, because of fluctuation of the statuses of deformation, it is necessary to use "the secondary calculation" to receive a plastic deformation field from the elasticity. Nevertheless it is necessary to notice, that the nature of process of plastic deformation is the noncommutative nature.

So, the process of deformation $P=\left\{E_{i j}(x, t)\right\}$ is given. It is a noncommutative deformation space. The deformation wave $E_{i j}(x, t)$ is a noncommutative wave. For complete descriptions of noncommutative wave we have to use the space being tensor product $C\left(R_{4}\right) \otimes M_{n}$, where $C\left(R_{4}\right)$ - is a algebra of smooth function determined on usual spacetime, and $M_{n}$ - is a algebra of $n \times n$ matrixes. In this general space the matrix function $E(x, t)$ looks like (for 2-dimension case): $E(x, t)=\left(\begin{array}{cc}A & B \\ C & D\end{array}\right) \in C\left(R_{4}\right) \otimes M_{2}$, where $A, B, C, D$ - is 1-form. Now our task is to construct a noncommutative deformation bundle on the $C\left(R_{4}\right) \otimes M_{2}$. This problem was performed in frameworks of noncommutative geometry Connes [9]. There are two important objects in this geometry. It is associative algebra $\mathcal{A}$ and universal algebra of the differential forms $\Omega_{D}^{1}(\mathcal{A})$ on the algebra $\mathcal{A}$. The deformation space $P=C\left(R_{4}\right) \otimes M_{n}$ plays role of associative algebra $\mathcal{A}$, at the same time universal algebra $\Omega_{D}^{1}(\mathcal{A})$ looks like $[10]$ :

$$
\Omega_{D}^{1}(\mathcal{A})=\Omega_{D}^{1}\left(C\left(R_{4}\right)\right) \otimes \Omega_{D}^{1}\left(M_{n}\right)
$$

It is tensor product of algebra of the differential forms over algebra of smooth function and algebra of the differential forms over algebra of $n \times n$ matrices. In turn, this algebra $\Omega_{D}^{1}(\mathcal{A})$ can be present through the direct sum of a horizontal and vertical part

$$
\Omega_{D}^{1}(\mathcal{A})=\Omega_{H}^{1} \oplus \Omega_{V}^{1}
$$

and

$$
\Omega_{H}^{1}=\Omega_{D}^{1}\left(C\left(R_{4}\right)\right) \otimes M_{n} ; \Omega_{V}^{1}=C\left(R_{4}\right) \otimes \Omega_{D}^{1}\left(M_{n}\right)
$$

Thus, differential calculation on algebra $\mathcal{A}$, being in usual external differential, is chosen. More precisely, if through $\operatorname{Der}(\mathcal{A})$ we had designated Lie algebra of derivative on $\mathcal{A}$, we shall receive

$$
\operatorname{Der}(\mathcal{A})=\left(\operatorname{Der}\left(C\left(R_{4}\right)\right) \otimes 1\right) \oplus\left(C\left(R_{4}\right) \otimes \operatorname{Der}\left(M_{n}\right)\right)
$$

Differential $d f$ of an element $f \in \mathcal{A}$ also is divided for the sum

$$
d f=d_{H} f+d_{V} f
$$

where $d_{H} f$ and $d_{V} f$ belong to $\Omega_{H}^{1}$ and $\Omega_{V}^{1}$ respectively. If $E_{k}, k \in\left\{1,2, \ldots, n^{2}-1\right\}$ is the basis of algebra $M_{n}$, then $\partial_{k}=a d\left(i E_{k}\right)$ is a basis of algebra $\operatorname{Der}\left(M_{n}\right)$ and $\left[\partial_{k}, \partial_{l}\right]=$ $\sum C_{k l m} \partial_{m}$. So, the basis $\theta^{k}$ of algebra $\Omega_{D}^{1}\left(M_{n}\right) \subset \Omega_{D}^{1}(\mathcal{A})$ is determined as $\theta^{k}\left(\partial_{l}\right)=\delta_{l}^{k} 1$. 
If through $\theta^{i}=\left(\theta^{\alpha}, \theta^{a}\right)$, where $\alpha=0,1,2,3 ; a \in\left\{1,2, \ldots, n^{2}-1\right\}$, the basis of algebra $\Omega_{D}^{1}(\mathcal{A})$ is designated, then the basis in $\operatorname{Der}(\mathcal{A})$ will be as $e_{i}=\left(e_{\alpha}, e_{a}\right)$ and $e_{\alpha}=e_{\alpha}^{\mu} \partial_{\mu}$ are 4-generators for algebra $C\left(R_{4}\right)$, and $e_{\alpha}=a d\left(E_{\alpha}\right)$ is a basis of $\operatorname{Der}\left(M_{n}\right)$. In this case $\theta^{i}$ accepts a kind

$$
\theta^{\alpha}=\theta_{\mu}^{\alpha} d x^{\mu}, \theta^{a}=E_{b} E^{a} d E^{b}
$$

Differential $d_{H}$ and $d_{V}$ will accept a kind

$$
d_{H} f=e_{\alpha} f \theta^{\alpha}, d_{V} f=e_{a} f \theta^{a}, f \in \mathcal{A} .
$$

Besides, there is an canonical element $\theta \in \Omega_{D}^{1}\left(M_{n}(C)\right)$ determined as $\theta=E_{k} \theta^{k}$. Within the framework of Connes geometry, noncommutative bundle is right, or left $\mathcal{A}$ - module, or more exact, is Hermit $\mathcal{A}$ - module. On this module the following connection is defined $[10]$

$$
\omega=A+\chi
$$

and $A$ - it is an element of $\Omega_{H}^{1}$ and $\chi$ - an element of $\Omega_{V}^{1}$. In turn, the element $\chi$ is divided for the sum

$$
\chi=\theta+\phi
$$

because of the fact, that $\Omega_{D}^{1}\left(M_{n}\right) \subseteq \Omega_{V}^{1}$. Here $\phi$ is a Higgs field. Higgs field $\phi$ may be the temperature field or disocation $[4,7]$. If $\mathcal{U}$ is the group of gauge maps, then an element $\omega, A, \theta, \phi$ will be transformed under action $g \in \mathcal{U}$ by a rule

$$
\begin{gathered}
\omega^{\prime}=g^{-1} \omega g+g^{-1} d g, \\
A^{\prime}=g^{-1}+g^{-1} d_{H} g, \theta^{\prime}=\theta, \phi^{\prime}=g^{1-} \phi g .
\end{gathered}
$$

The intensity of a field of deformation is determined through the 2-form $\Omega$

$$
\Omega=d \omega+\omega^{2},
$$

or

$$
\Omega=F+D_{H} \phi+\Omega_{V}
$$

and

$$
\begin{aligned}
& F=d_{H} A+[A, A]=\frac{1}{2} F_{\alpha \beta} \theta^{\alpha} \theta^{\beta} \\
& \Omega_{V}=\frac{1}{2} \Omega_{a b} \theta^{a} \wedge \theta^{b}, \quad \Omega_{a b}=\left[\phi_{a}, \phi_{b}\right]-C_{a b}^{c} \phi_{c} \\
& D_{H} \phi=d_{h} \phi+A \phi+\phi A=\theta^{\alpha}\left(e_{\alpha}+\left[A_{\alpha}, \phi\right]\right) \\
& \phi=\phi_{\alpha} \theta^{\alpha}, A=A_{\alpha} \theta^{\alpha}
\end{aligned}
$$

Action of the deformation field will be as

$$
L=\frac{1}{2}\left(\Omega_{i j}, \Omega_{i j}\right)
$$

Thus, in frameworks of our model [7], we shall receive probability of transition to plasticity

$$
Z=\int \exp (-k L)
$$


where $k$ - is a factor of elasticity.

\section{THE PLASTIC INSTANTON}

We discuss the problem put above. For simplicity we are limited to consider only plane deformation and noncommutative wave $E(x, t)$ is considered in the condition: $A=$ $D \neq 0, C=B=0$. Actually, all above mentioned models were considered only in such similar situations. In these models one assumed about the existence of limiting surfaces of deformations (or loading). It is equivalent to the requirement of finiteness of action of deformation fields. Then, for search of a configuration of a deformation field, it is necessary to solve the equation, which is a extreme condition of action $L$,

$$
\delta L\left[A_{\mu}\right]=0
$$

However, we have also other extreme condition for action

$$
-\int d^{4} x \operatorname{Tr}\left[\left(F_{\mu \nu}+ \pm \tilde{F}_{\mu \nu}\right)^{2}\right]
$$

where $\tilde{F}_{\mu \nu}$ is dual with $F_{\mu \nu}$. Because of that $\operatorname{Tr}\left[F_{\mu \nu}, F_{\mu \nu}\right]==\operatorname{Tr}\left[\tilde{F}_{\mu \nu}, \tilde{F}_{\mu \nu}\right]$, we receive

$$
-\int d^{4} x \operatorname{Tr}\left[F_{\mu \nu}, F_{\mu \nu}\right] \geq \mp \int d^{4} x \operatorname{Tr}\left[\tilde{F}_{\mu \nu}, \tilde{F}_{\mu \nu}\right],
$$

it like as in work of Belavin etc. [12], we are interested in importance of self-dual and self-antidual configuration of a deformation field. Thus, one of a extreme condition of action will be writter write down as

$$
\tilde{F}_{\mu \nu}=\mp F_{\mu \nu} .
$$

Belavin etc. used this condition for giving the instanton of gauge field. So, instead of the equation (3.1) we shall solve a more simple equation (3.2). We use method in [11] for search of multi-instanton in special cases of plane deformation, when $n, r=1$. Let the field of deformation $A=A_{\mu} d x^{\mu} \in \Omega_{D}^{1}\left(R_{4}\right) \otimes 1$, have an individual kind of

$$
A_{\mu}(x)=i B_{\mu \nu} \partial_{\nu}(\ln \phi(x))
$$

where $\phi(x)$ - is a scalar function, and the matrix $B_{\mu \nu}$ looks like

$$
B_{\mu \nu}=\frac{1}{2}\left\{\begin{array}{cccc}
0 & +\sigma_{3} & -\sigma_{2} & -\sigma_{1} \\
-\sigma_{3} & 0 & +\sigma_{1} & -\sigma_{2} \\
+\sigma_{2} & -\sigma_{1} & 0 & -\sigma_{3} \\
+\sigma_{1} & +\sigma_{2} & +\sigma_{3} & 0
\end{array}\right\},
$$

where $\sigma_{1}, \sigma_{2}, \sigma_{3}$ - are Pauli matrices. The gauge group is admitted as $S U(2)$. Setting $A_{\mu}$ in expression (2.2), the condition of self-dual (3.2) will be as

$$
\partial_{\sigma} \partial_{\sigma}(\ln \phi)+\left(\partial_{\sigma} \ln \phi\right)^{2}=0
$$


or

$$
\frac{\nabla^{2} \phi}{\phi}=0, \text { where } \nabla^{2}=\partial_{\sigma} \partial_{\sigma}
$$

Using the method in [12] we receive the instanton, being the solution of equation (3.4):

- instanton

$$
\phi(x)=1+\frac{\lambda_{1}^{2}}{|x|^{2}}
$$

- multi-instanton

$$
\phi(x)=1+\sum_{i=1}^{N} \frac{\lambda_{i}^{2}}{\left|x_{\mu}-a_{i \mu}\right|^{2}}
$$

where $a_{i \mu}, \lambda_{i}$ - is any valid constant. Setting $\phi(x)$ from (3.5) and (3.6) into (3.3) we receive the deformation wave as matrices:

- one-instanton

$$
F_{\mu}(x)=-2 i \lambda^{2} B_{\mu \nu} \frac{y_{\nu}}{y^{2}\left(y^{2}+\lambda^{2}\right)}
$$

multi-instanton

$$
A_{\mu}(x)=-2 i B_{\mu \nu}\left(\sum_{i}^{N} \frac{\lambda_{I}^{2} y_{i \nu}}{y_{i}{ }^{4}}\right) /\left(1+\sum_{j}^{N} \frac{\lambda_{i}^{2}}{\left|y_{j}\right|^{2}}\right),
$$

where $\left(y_{i}\right)_{\mu} \equiv\left(x-a_{i}\right)_{\mu}, \quad, \mu=1,2, \ldots, N$.

\section{TOPOLOGICAL CHARACTER OF KOITER SINGULARITY}

What is the real nature of Koiter sector on the limiting surface of deformation. With the account of solution of instanton, this problem can be easily clear. Really, using this instanton, we receive topological Pontriagin number

$$
Q=-\frac{1}{16 \pi^{2}} \int d^{4} x \operatorname{Tr}\left[F_{\mu \nu} F_{\mu \nu}\right]
$$

This number tells us, that during the process of plastic deformation the space of deformation will be divided on $Q$ independent sectors. Thus, limiting the surface of deformation, constructed in this space, is divided on $Q$ parts, too. It is Koiter sectors described in works [1-3], [13-14]. We see, that the occurrence of Koiter singularity has the topological reasons. Now we can say, the process of plastic deformation is a process of birth and distribution of solitons and like-solitons. It is necessary to notice, that the defect, from the point of view of fluctuation, is one of kinds of solitons. The soliton wave is considered as the concentrated located energy, which is distributed in space without dissipation with constant velocity. Thus, it will allow us to understand, why the plastic part of the stress - deformation diagram has the horizontal form.

\section{REFERENCES}

1. A. A. Myushin, Mechanics of Continuous Medium, MGU 1990.

2. Iu. N. Rabotnov, Mechanics of a Deformable Body, M.:Science 1988. 
3. V. D. Kliushnikov, Physical and Mathematical Basis of Elasticity and Plasticity, MGU 1994.

4. V. K. Trinh, Noncommutative thermomechanical deformation, Physics Bulletin of Moscow University 57 (1) (2002) 49-51.

5. V. K. Trinh, A model of a crack in the mechanism of dynamical phase transition and the physical sense of Kachanov's function, Fiz. Mezomechanic 5 (4) (2002)

6. V. K. Trinh, A model of a crack in the vicinity of phase transition, Doklady Physics (English translation of Doklady Akademii Nauk) $\mathbf{4 5}$ (6) (2000).

7. V. K. Trinh, Noncommutative plastic deformation and the essence of the Ilyushin's isotropy postulate, Doklady Physics (English translation of Doklady Akademii Nauk) 46 (5) (2001) 373.

8. M. Ausloos, Phase transitions theory approach to fracture of materials, Solid Stat. Comm. 59 (6) (1986) 401-404.

9. A. Connes, Noncommutative differential geometry, Publ. I. H. E. S. 62 (1989) 257.

10. M. Daboit-Violette, R. Kerner, J. Madore, Noncommutative differential geometry and new modes of gauge theory, J. Math. Phys. 31 (2) (1990) 323.

11. R. Rajaraman, Soliton and Instanton in the Quantum Theory of a Field, M.: World, 1985.

12. A. A. Belavin, A. M. Polyakov, A. S. Schwartz, Yu. S. Tyupkin, Pseudoparticle solution of the Yang-Mils equation, Phys. Lett. 59B (1975) 85.

13. L. I. Sedov, Mechanics of Continuous Medium, M. Science, 1973.

14. W. T. Koiter, Stress-strain relations, uniqueness and variational theorems for elasticplastic materials with a singular yield surface, Quart. App. Math. 11 (3) (1953) 350354.

15. A. A. Katsneson, Introduction into Solid State Physics (in Russian), Moscow, 1984.

16. H. Li and N. Chandra, Int. J. Plas. 1 (6) (2003) 849.

17. S. Dachkovski and M. Boohm, Finite thermoplasticity with phase charge base on isomorphifsm, Int. J. Plast. 1 (6) (2003) 699.

18. Ch. Tsakmakis, Arch. Mech., Remark on the postulate of Ilyshin Int. J. Plast. (in press) 49 (1997) 677.

19. Ch. Tsakmakis, Arch. Mech., Description of plastic anisitropy effects at large deformations - part I: Restriction imposed by the second law and the postulate of Ilyushin, Int. J. Plast. 54 (2001) 520.

Received May 20, 2005

\section{BIẾN DẠNG KHÔNG GIAO HOÁN VÀ BẢN CHẤT TOPO CƯA CÁC KỲ DỊ KOITER}

Trong bài báo này chúng ta xây dựng mô hình biến dạng dẻo không giao thoa hoán và chứng minh giả thiết Koiter. Chúng ta đã chỉ ra rằng sự xuất hiện các điển kỳ dị Koiter có nguyên nhân topo và các điểm kỳ dị Koiter là số topo Pontriagin. 